\title{
Ablation characteristics of quantum square pulse mode dental erbium laser
}

Nejc Lukač

Tomaž Suhovršnik

Matjaž Lukač

Matija Jezeršek

\section{SPIE.}




\title{
Ablation characteristics of quantum square pulse mode dental erbium laser
}

\author{
Nejc Lukač, ${ }^{a, *}$ Tomaž Suhovršnik, ${ }^{b}$ Matjaž Lukač, ${ }^{c}$ and Matija Jezeršek ${ }^{a}$ \\ aUniversity of Ljubljana, Faculty of Mechanical Engineering, Askerčeva 6, Ljubljana 1000, Slovenia \\ bUniversity of Ljubljana, Faculty of Physics, Jadranska 39, Ljubljana 1000, Slovenia \\ 'Institute Josef Stefan, Jamova 39, Ljubljana 1000, Slovenia
}

\begin{abstract}
Erbium lasers are by now an accepted tool for performing ablative medical procedures, especially when minimal invasiveness is desired. Ideally, a minimally invasive laser cutting procedure should be fast and precise, and with minimal pain and thermal side effects. All these characteristics are significantly influenced by laser pulse duration, albeit not in the same manner. For example, high cutting efficacy and low heat deposition are characteristics of short pulses, while vibrations and ejected debris screening are less pronounced at longer pulse durations. We report on a study of ablation characteristics on dental enamel and cementum, of a choppedpulse Er:YAG [quantum square pulse (QSP)] mode, which was designed to reduce debris screening during an ablation process. It is shown that in comparison to other studied standard Er:YAG and Er,Cr:YSGG laser pulse duration modes, the QSP mode exhibits the highest ablation drilling efficacy with lowest heat deposition and reduced vibrations, demonstrating that debris screening has a considerable influence on the ablation process. By measuring single-pulse ablation depths, we also show that tissue desiccation during the consecutive delivery of laser pulses leads to a significant reduction of the intrinsic ablation efficacy that cannot be fully restored under clinical settings by rehydrating the tooth using an external water spray. ๑ 2016 Society of Photo-Optical Instrumentation Engineers (SPIE) [DOI: 10.1117/1.JBO.21.1.015012]
\end{abstract}

Keywords: laser ablation; hard dental tissue; erbium lasers; water cooling; triangulation; hydrokinetic effect.

Paper 150663R received Oct. 5, 2015; accepted for publication Dec. 18, 2015; published online Jan. 25, 2016.

\section{Introduction}

Pulsed midinfrared erbium lasers, such as such Er:YAG (with a wavelength of $\lambda=2.94 \mu \mathrm{m})$ or Er,Cr:YSGG $(\lambda=2.78 \mu \mathrm{m})$, have been recognized as the lasers of choice when fast ablation of human tissues with minimal side effects is required. ${ }^{1-4}$ This is because erbium laser wavelengths approximately coincide with the major absorption peak of the water molecule and are thus strongly absorbed in virtually all biological tissues. ${ }^{4}$ Numerous studies ${ }^{2-8}$ have established that thermal cutting is the basic mechanism by which tissue is removed with erbium lasers. The thermal cutting mechanism is based on heating the interstitially trapped water within tissue to the evaporation temperature. $^{3}$ This leads to explosive subsurface expansion of interstitially trapped water inside the tissue and subsequently to microexplosive internal tearing of the tissue. Erbium lasers are because of their high ablation efficacy, used in various medical fields including dentistry, dermatology, surgery, ophthalmology, and gynecology. $2,9-12$

In order to perform minimally invasive medicine, it is important to optimize erbium laser parameters such that laser cutting is fast, cuts are sharp and precise, the procedure is quiet and with minimal vibrations imposed on the treated tissue, and the amount of residual heat that remains in the tissue following erbium laser irradiation is minimal. However, with standard, bell or quasisquare-shaped erbium pulse modes,${ }^{13}$ these requirements are to a certain extent contradictory, as parameters that may be optimal, e.g., achieving fastest ablation, may not be optimal when minimal vibrations and noise are required. ${ }^{14,15}$
For example, it is well understood that ablation thresholds decrease toward shorter pulse durations, ${ }^{16-19}$ where short pulses are pulses shorter than the thermal relaxation time of the ablated tissue. ${ }^{18,19}$ At shorter times, the energy has little time to escape from the ablated volume, and so less heat is diffused into the surrounding tissue. ${ }^{18}$ Shorter pulses, therefore, result in faster ablation and smaller amount of residual heat deposition. ${ }^{20}$ On the other hand, shorter laser pulses are expected to produce higher-frequency tissue vibrations ${ }^{21}$ and may therefore cause more discomfort to patients. ${ }^{22}$

Similarly, when undesirable interaction of the laser beam with the debris cloud is considered, ${ }^{23}$ the effects of debris screening are less pronounced at longer pulse durations. ${ }^{18}$ At short pulse durations, the incoming laser beam gets strongly absorbed in the dense debris cloud, which forms above the irradiated area, resulting in a reduced ablation rate. Cuts are also less precise, since the scattering effect caused by the cloud leads to spreading of the laser beam. ${ }^{24-26}$ Finally, the laserreheated debris cloud is expected to contribute, as it falls back to the tissue surface, to additional heating of the tissue, and consequently to additional residual heat deposition. ${ }^{25}$

Recently, a special quantum square pulse (QSP) modality has been proposed with a goal of reducing the undesirable residual side effects of erbium laser beam scattering and absorption in the debris cloud. ${ }^{24}$ The QSP mode functions by chopping a standard laser pulse of longer duration $(\sim 600 \mu \mathrm{s})$ into a series of five short pulses of $50 \mu \mathrm{s}$ separated by $85 \mu \mathrm{s}$. This makes the QSP mode ablate with efficacy of short pulses, with the benefit of reduced debris screening at longer pulses. This is due to the 
fact that the duration of each of the pulse quanta $(\sim 50 \mu \mathrm{s})$ is shorter than the rise time of the debris cloud, while the separation between the pulse quanta of $\sim 85 \mu$ s is longer than the decay time of the debris cloud. Ablation measurements in dental enamel have subsequently demonstrated that as a result of reduced undesirable effects of laser-debris interaction, the use of the QSP mode results in sharper cuts and higher ablation efficacy in enamel. ${ }^{23,25,26}$

In this paper, we report on a systematic comparison of ablation characteristics of standard erbium laser pulse modes of different pulse durations and wavelengths (Er:YAG and Er,Cr:YSGG) and of the new Er:YAG QSP chopped pulse profile mode. On an example of ablation in dental enamel and cementum, we measured ablation characteristics of the QSP mode with respect to ablation drilling efficacy, vibrations, and residual heat deposition.

Typically, in order to improve the measurement accuracy, ablation cavities are made with a series of consecutive pulses, and the ablation efficacy expected from a single pulse is calculated by dividing the measured volume or depth by the number of delivered pulses. ${ }^{5,27}$ However, this approach may not be always appropriate since, especially for tissues with relatively low water content, the ablation efficacy has been observed to get progressively reduced upon consecutive delivery of laser pulses. ${ }^{28,29}$ When using such cumulative ablation methods, the measurement process influences the ablation process, which is being studied, and therefore, erroneous results and conclusions may be reached. For example, it is well known that for dental enamel, the ablation process completely stalls after a consecutive delivery of $\sim 10$ laser pulses, unless the enamel is cooled by an external water spray..$^{27,30-38}$ To explain this observation, an additional "hydrokinetic" ablative mechanism has been proposed, which is based on the reception by water spray particles of the laser energy and consequent "impartation of disruptive forces" by accelerated water particles to the tissue. ${ }^{32,33}$ The actual mechanism of laser ablation in the presence of water spray is still controversial to a certain extent. ${ }^{27,34,35}$

In our study, in order to evaluate the influence of cumulative desiccation on measured ablation efficacy, we carried out not only cumulative but also individual pulse ablation measurements, using a sensitive triangulation measurement technique. We show that on enamel, the initial, "intrinsic" drilling efficacy (in $\mathrm{mm}^{3} / \mathrm{J}$ ) of the first pulse in a sequence of pulses is about two-times higher compared to the cumulative efficacy of 10 consecutively delivered pulses.

\section{Materials and Methods}

The Er:YAG system used was a LightWalker AT (manufactured by Fotona), and the Er,Cr:YSGG laser was a WaterLase iPlus (manufactured by Biolase). Both lasers were flashlamp pumped and were fitted with the appropriate noncontact handpieces (Fotona H02 handpiece and Biolase Turbo handpiece with MX11 tip).

The lasers were operated in the following pulse modes and durations: (a) Er:YAG laser in standard super short pulse (SSP, $90 \mu \mathrm{s}$ ), medium short pulse (MSP, $130 \mu \mathrm{s}$ ), short pulse (SP, $220 \mu \mathrm{s}$ ), and long pulse (LP, $360 \mu \mathrm{s}$ ) pulse modes, and in the new QSP mode $(600 \mu \mathrm{s})$ and (b) Er,Cr:YSGG laser in $H$ pulse $(400 \mu \mathrm{s})$ duration mode. The temporal pulse shapes of the above modes have been reported in Refs. 17, 26, and 39, and are different for different modes and erbium laser types. For this reason, a more meaningful comparison of the measured output laser pulse shapes can be made by integrating the delivered laser energy over the duration of the laser pulse. ${ }^{39}$ The above pulse durations represent times during which $90 \%$ of the cumulative laser pulse energy $(300 \mathrm{~mJ}$ for Er:YAG, and $170 \mathrm{~mJ}$ for Er,Cr:YSGG) were delivered to a target. The temporal structure of the chopped QSP mode and its relation to the development of ablation debris cloud is shown in Fig. 1.

Approximately 600- $\mu$ s long QSP macropulse consists of five approximately $t_{\mathrm{QSP}} \approx 50 \mu \mathrm{s}$ long micropulses (pulse quanta) separated by a temporal spacing of $\Delta t_{\mathrm{QSP}} \approx 85 \mu \mathrm{s}$. The duration of QSP micropulses is shorter than the rise time of the debris cloud, while the separation between the micropulses is longer than the decay time of the debris cloud. ${ }^{24}$ It should be noted here that the duration of the QSP micropulses is also shorter than the thermal relaxation time $(\tau)$ for hard dental tissues of approximately $\tau=110 \mu \mathrm{s} .{ }^{18}$

The experiments were conducted on randomly chosen extracted premolar and molar teeth, which were stored in a physiological saline solution immediately following extraction. Before each ablation experiment, the tooth was positioned to have its surface perpendicular to the laser beam and to be at focal distance of the laser beam. The diameters $(d)$ of the laser beam at the focal point were approximately $d=0.9 \mathrm{~mm}$ for the Er:YAG laser and $d=0.7 \mathrm{~mm}$ for the Er,Cr:YSGG laser, with exact diameters measured for each modality and laser energy using a sensitive photographic paper.
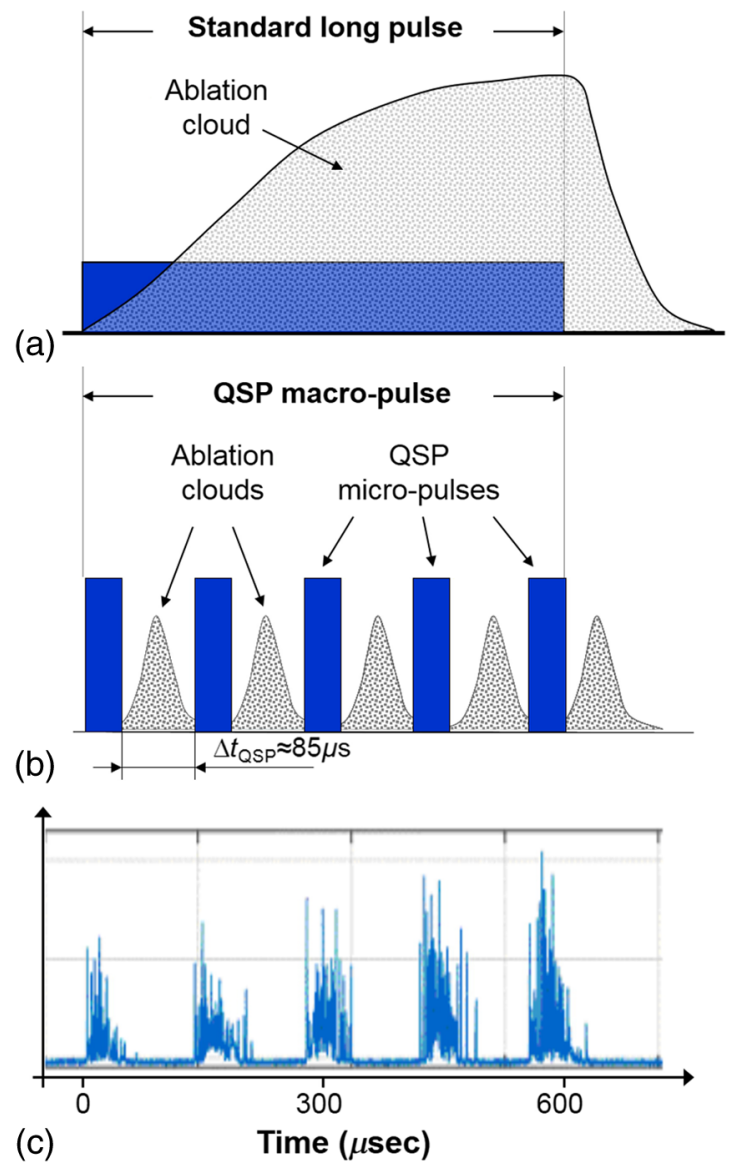

Fig. 1 (a) Standard laser pulse; (b) QSP laser pulse. A 600- $\mu$ s long macropulse is chopped into five micropulses with micropulse duration of $t_{\mathrm{QSP}}=50 \mu \mathrm{s}$, and temporal spacing between the micropulses of $\Delta t_{\mathrm{QSP}}=85 \mu \mathrm{s}$. 
Measurements were made on enamel, which covers the crown of the tooth, and on cementum, which covers the root of the tooth. Enamel and cementum were chosen in order to evaluate the effects of Er:YAG laser radiation on hard tissues at both extremes, i.e., in (a) enamel, which has a high content of hydroxyapatite $(96 \%)$ and a very low intrinsic water content $\left(3 \%_{\text {weight }}\right)$ and (b) in cementum, which consists of $45 \%$ of hydroxyapatite, $33 \%$ of organic material mainly collagen and protein polysaccharides and $22 \%$ weight water. $^{40}$ For comparison, dentin contains $12 \%_{\text {weight }}$ of water, bone contains $\sim 22 \%_{\text {weight }}$ of water, and soft tissue contains $70 \%_{\text {weight }}$ of water.

Single-pulse laser energy, $E_{\text {out }}$ (in $J$ ), was measured with an external energy meter at the handpiece output. Special care was taken to ensure that with both laser systems, the ablation measurement pulse sequence was emitted after the laser output had already stabilized. The lasers were operated at a constant repetition rate of $2 \mathrm{~Hz}$ and the repetition rate $(f)$ of the pulses delivered to the tissue was controlled using an external shutter. Unless otherwise stated, the repetition rate was set to $f=0.2 \mathrm{~Hz}$. Since following an ablative Er:YAG laser pulse the ablated tooth surface returns to the initial temperature in $\sim 0.5 \mathrm{~s},{ }^{20}$ this slow repetition rate ensured that the tooth surface cooled down between pulses, and therefore, each pulse started the ablation process from the same initial tooth temperature.

With the Er:YAG laser, $200 \mathrm{~mJ}$ of laser pulse energy was used on cementum and $300 \mathrm{~mJ}$ on enamel. Different energies were chosen in order to obtain cavities of similar depth in both types of tissues. Similarly, with the smaller beam diameter Er,Cr:YSGG laser, lower pulse energies of 113 and $170 \mathrm{~mJ}$ were used on cementum and enamel, respectively. The single-pulse laser fluence $\left[F\right.$ (in $\left.\left.\mathrm{J} / \mathrm{mm}^{2}\right)\right]$, averaged over the irradiated area at the focal point, was calculated from $F=E_{\text {out }} /\left(\pi d^{2} / 4\right)$.

All laser pulses under consideration were multimode (transverse and longitudinal) pulses. The Er:YAG laser intensity distributions in the irradiated area were approximately flat-top, concluding from the observed shapes of the shallow ablation cavities $(h<0.1 \mathrm{~mm})$ made with a single-laser pulse. No effort was made to measure exact intensity distributions as it was determined in a separate study. ${ }^{41}$ The study showed that the shape of the ablated hole (with diameter smaller than $1 \mathrm{~mm}$ ) depends not only on the spatial laser intensity distribution but also on the depth of the ablated hole. Holes of medium depth (up to $h \approx 0.5 \mathrm{~mm}$ ) are approximately conical while deeper cavities obtain a more cylindrical shape. ${ }^{41}$

The irradiation fluences $F$, for QSP, SSP, SP, and $\mathrm{H}$ modes, as calculated from the measured laser pulse energies and beam diameters, were correspondingly $38,35,40$, and $44 \mathrm{~J} / \mathrm{cm}^{2}$ on enamel, and $26,24,27$, and $29 \mathrm{~J} / \mathrm{cm}^{2}$ on cementum. These fluences were well above the published ablation threshold fluences $F_{\text {th }}$ for enamel (with low water content) and dentin (with high water content) of $F_{\text {th }}=3.5$ and $4 \mathrm{~J} / \mathrm{cm}^{2}$, respectively. ${ }^{18}$

The single-pulse ablation drilling efficacy [DF (in $\mathrm{mm}^{3} / \mathrm{J}$ )], defined as ablation depth per laser fluence, was calculated from $\mathrm{DF}=L /(N \times F)$, where $L$ is the depth of the ablated hole following $N$ consecutively delivered laser pulses of the same pulse energy to the same spot on a target. Note that the drilling efficacy can be considered to represent also the ablation efficacy $^{35}$ [AE (in $\mathrm{mm}^{3} / \mathrm{J}$ )], defined as ablated volume per laser energy, assuming a cylindrically shaped hole having a constant diameter d over the depth $L$ of the hole. In reality, the diameter of the hole gets smaller toward the bottom of the ablated cavity, ${ }^{41}$ and therefore, the actual ablation efficacy is expected to be smaller.

Experiments were carried out under three conditions: (a) "dry" conditions where after the tooth has been taken out of the saline solution, no external water was added to the tooth during the ablation experiment; (b) "hydration" experimental setup, which was designed to prevent, at least to a certain extent, the desiccation of the irradiated tooth surface. This was accomplished by rehydrating the ablation area following each laser pulse with an external water spray. This "hydration" phase lasted for $T=1 \mathrm{~s}$ following each laser pulse. After rehydration, a special care was taken that the tooth surface was left devoid of any surface water. An air flow was directed to the ablation area for the duration of an "air-blowing" phase lasting $4 \mathrm{~s}$, at the end of which the tooth was again irradiated with a subsequent laser pulse. Note that this "hydration" experimental setup was similar to the "pores" condition used in Ref. 35; and (c) "spray" conditions where during the ablation experiment, a water/air spray was delivered continuously to the tooth through the corresponding laser system handpieces. The spray water flow rate (in $\mathrm{ml} / \mathrm{min}$ ) was determined by measuring the time and water volume collected in an external container during that time. Unless otherwise stated, the error bars included in the figures represent standard deviations of the sample data.

\subsection{Optical Microscope Measurements}

When optical microscope was used to measure the depth $(h)$ of ablated holes, each ablation cavity was made with $N=10,20$, or 30 consecutive laser pulses of the same pulse energy delivered to the same spot. The depth of the ablated cavity following the ablation pulse sequence was then measured using a focusing optical microscope (Leica, M205C). Each data point represents an average obtained from 10 different cavities, each made with $N$ consecutive pulses.

\subsection{Laser Triangulation Profilometry}

Depth measurements of ablated holes during drilling were based on laser triangulation profilometry. ${ }^{35,42}$ Figure 2 shows the experimental system where the laser triangulation is arranged on the right side. The tooth was attached on a three-axis translation stage (TS) with epoxy. Erbium laser pulses were delivered through a handpiece (LH), which was fixed at a constant distance from the TS. A laser line projector (LLP) was used to generate a vertical light plane, which intersected the middle of the hole and the surrounding area (StockerYale, model Lasiris SNF, $10 \mathrm{~mW}, \lambda=670 \mathrm{~nm}$, line thickness $0.1 \mathrm{~mm}$ ). The light plane was coincident with the optical axis of the LH, but the LLP was tilted around the horizontal axis 15 deg to prevent shadowing from the LH. The cross-section between the laser plane and the illuminated area was recorded with a digital camera (Nikon D610) with a macrolens (Nikkor Micro 200 f/4D). Since the camera was tilted against the laser plane at a triangulation angle (30 deg), the laser contour on the acquired image has a similar shape as the cross-section of the hole.

Images were acquired after each laser pulse and transferred to a personal computer (PC), where the profile was extracted using a subpixel peak detection algorithm. The depth of the hole was measured as a height difference between the nonablated and the ablated region. Depth transformation from image $(v)$ into metric units $(z)$ was based on the known optical magnification $(m=-1)$, pixel size $(\Delta=6 \mu \mathrm{m})$, and triangulation angle 


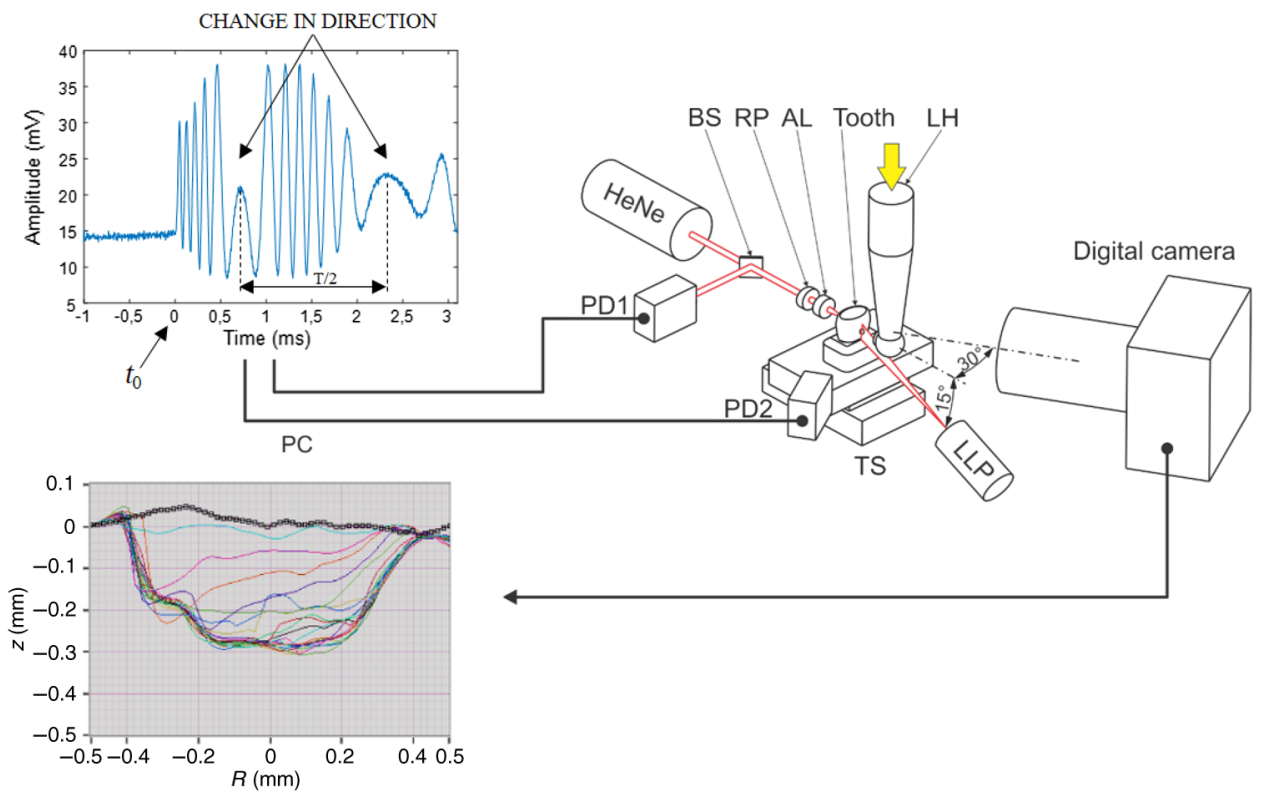

Fig. 2 Experimental setup for measurements of hole depth and tooth vibrations during laser drilling. LLP, digital camera, and PC are used for laser triangulation. HeNe laser, BS, RP, AL, two photodetectors (PD1 and PD2), and oscilloscope are used for interferometric measurement. The laser was fitted with a noncontact handpiece (LH). The graph in the top left shows a typical interferometer signal with $t_{0}$ denoting the beginning of the Er:YAG laser pulse and $T / 2$ being half the period of the measured vibration.

( $\alpha=30 \mathrm{deg})$ and was calculated from $z=\Delta v / m \sin \alpha$. An example of measured profiles after each of 20 consecutive laser pulses is shown in Fig. 2 within the PC's box.

\subsection{Interferometric Vibration Measurements}

An interferometric setup was implemented for measuring vibration amplitudes of the tooth during the laser drilling. The interferometer was based on the Fizeau configuration ${ }^{43}$ and is schematically shown on the left side of Fig. 2. A collimated laser beam from a HeNe laser $(\lambda=633 \mathrm{~nm}$, beam diameter $=$ $0.8 \mathrm{~mm}$ ) was guided through a $50 \%$ beam splitter (BS) and a wedge shaped reference plate (RP) and focused on the backside of the tooth by an achromatic lens (AL) with a focal length of $7.5 \mathrm{~mm}$. Interference between the reference beam, reflected from the RP, and the measuring beam, reflected from the tooth surface, was measured by a Si photodetector (PD1) $(10-\mathrm{MHz}$ bandwidth) positioned near the BS. In front of the PD1, a pinhole with diameter of $0.5 \mathrm{~mm}$ was attached which transmitted only the light from the central interference fringe. The second, InAs, photodetector (PD2) (60-MHz bandwidth) measured the reflected Er:YAG laser light and was used for triggering the oscilloscope (LeCroy, US, $600 \mathrm{MHz}$ Wave Runner 64MXi-A). The amplitude of the first tooth oscillation after each laser pulse (which occurs at $t_{0}$ ) was measured by counting the peaks and valleys on the measured signal between $t_{0}$ and the moment where the change in moving direction occurs. A typical example of such a change in moving direction is shown on oscilloscope in Fig. 2. The measured resonance frequency of the experimental setup was $\sim 300 \mathrm{~Hz} . T / 2$ in Fig. 2 represents half of the oscillation period, from the maximum displacement of the tooth in the first direction to the maximum displacement of the tooth in the opposite direction.

\section{Results}

\subsection{Optical Microscope Technique Measurements}

Figure 3 shows measured ablation depths in enamel for the Er: YAG (SSP) pulse duration mode for "dry" and "hydration" conditions. As can be seen from Fig. 2, under dry conditions, the ablation process on enamel stops after approximately $N=10$ consecutive pulses, with the maximal depth of the hole being limited to an "ablation saturation depth," $L_{\text {sat }}$. On the other hand, hydration conditions prevent ablation stalling, and the drilling process continues also for a larger number of pulses.

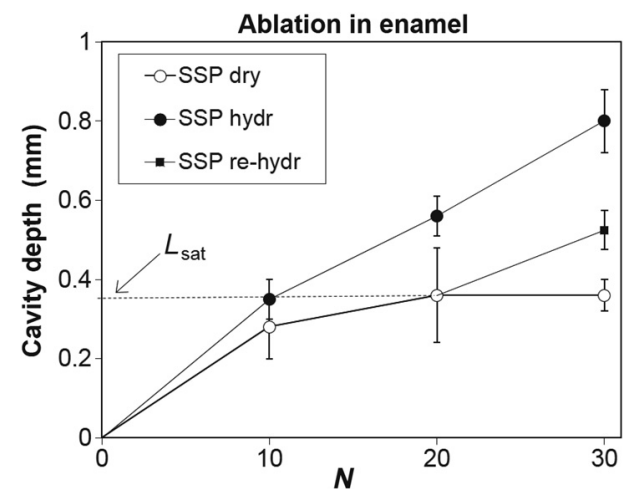

Fig. 3 Measured ablation depths in enamel for the Er:YAG (SSP) pulse duration mode with single-pulse energy of $300 \mathrm{~mJ}$ under "dry" (open circles) and "hydration" (full circles) conditions, as a function of the number of consecutively delivered laser pulses. Ablation depths were measured using an optical microscope technique. Under "dry" conditions, the ablation process stops at the saturation depth $L_{\text {sat }}$. The full square represents the ablation depth as obtained when the enamel was rehydrated following 20 consecutive pulses under "dry" conditions. 


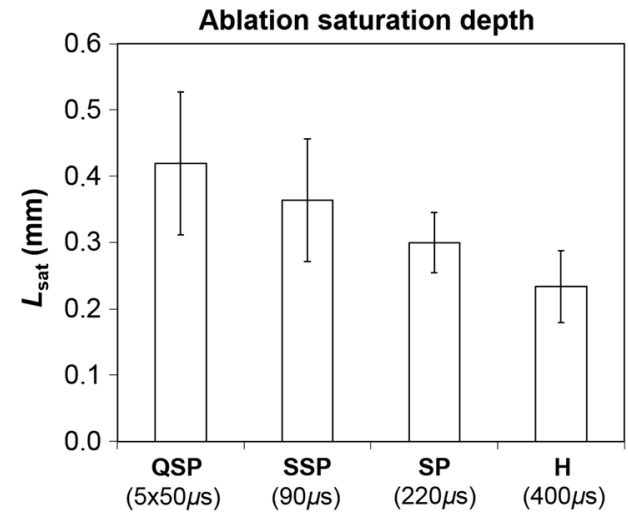

Fig. 4 Dependence of the ablation saturation depth $\left(L_{\text {sat }}\right)$ in enamel on the erbium laser pulse mode.

In a rehydration experiment represented in Fig. 3 by a full square, the enamel was first irradiated with $N=20$ pulses under "dry" conditions and then rehydrated with a water spray for $30 \mathrm{~min}$. After blowing off any remaining surface water, the same ablation cavity was irradiated with additional 10 pulses, again under "dry" conditions. Rehydrating of the "dried out" enamel was observed to restart the ablation process.

Ablation stalling was observed for all measured laser modes, with the depth $L_{\text {sat }}$ depending on the laser mode (see Fig. 4). The ablation saturation depth was measured to be largest for the QSP mode.

Figure 5 shows measured ablation depths in enamel for different pulse modalities under hydrated conditions. The ablation data were normalized to the same laser fluence of $35 \mathrm{~J} / \mathrm{cm}^{2}$ in order to be able to compare the ablation depths of various modalities in enamel, as would be obtained using exactly the same laser fluences. Here, it was assumed that within the small range of experimentally applied laser fluences (35 to $44 \mathrm{~J} / \mathrm{cm}^{2}$ ), which are significantly above ablation threshold, the dependence of the ablation depth on fluence for enamel is linear, in agreement with other published studies. ${ }^{5}$ As can be seen from Fig. 5, among all tested erbium modes under hydration conditions, the QSP mode exhibits largest drilling efficacy in enamel.

Measured ablation depths in cementum for the Er:YAG (SSP) pulse duration mode for "dry" and "hydration" conditions are depicted in Fig. 6. Measurements demonstrated that on

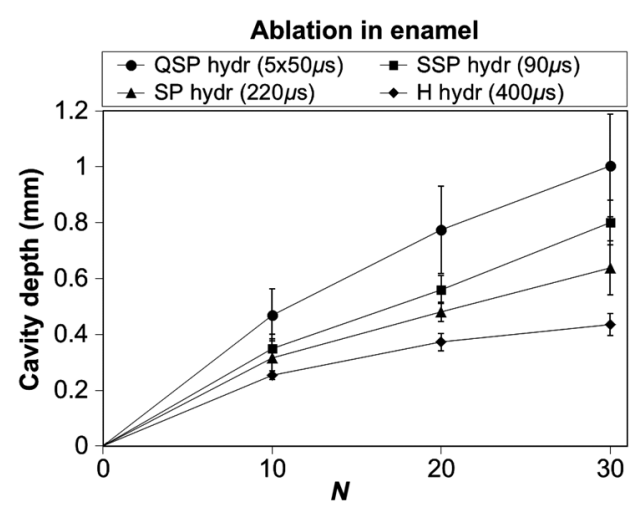

Fig. 5 Measured ablation depths in enamel normalized to the same laser fluence of $F=0.35 \mathrm{~J} / \mathrm{mm}^{2}$, under "hydration" conditions for different erbium laser modes. Ablation depths were measured using a focusing optical microscope.

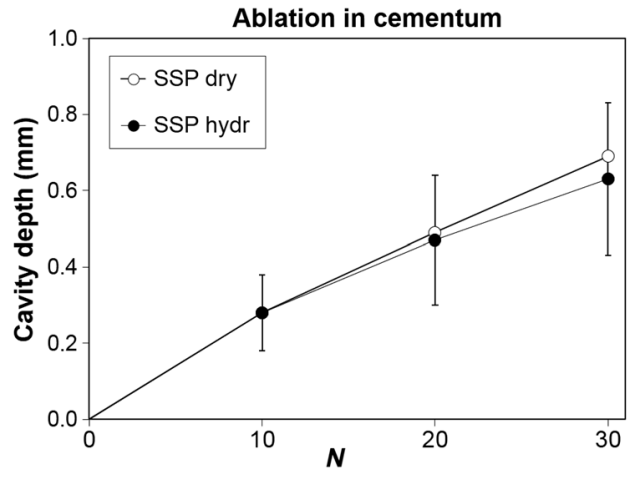

Fig. 6 Measured ablation depths in cementum for the Er:YAG (SSP) pulse duration mode with single pulse energy of $200 \mathrm{~mJ}$ under "dry" (open circles) and "hydration" (full circles) conditions, as a function of the number of consecutively delivered laser pulses. Ablation depths were measured using a focusing optical microscope technique.

cementum, there is no significant difference in observed cavity depths under "dry" and "hydration" conditions. The same observation was made not only for the SSP mode but also for all other erbium modes. Contrary to measurements on enamel, no significant ablation stalling is observed on cementum under "dry" conditions.

Figure 7 shows measured ablation depths in cementum for different pulse modalities under "dry" conditions. As in Fig. 5 for enamel, the ablation data were normalized to the same laser fluence of $35 \mathrm{~J} / \mathrm{cm}^{2}$ in order to be able to compare the ablation depths of various modalities in cementum and enamel, as would be obtained using exactly the same laser fluences. Again, this is assuming that within the small range of considered laser fluences ( 26 to $35 \mathrm{~J} / \mathrm{cm}^{2}$ ), the dependence of the ablation depth on fluence in cementum is linear. ${ }^{5}$ Similarly to measurements on enamel, the QSP mode exhibits largest drilling efficacy in cementum under "dry" and as well under "hydration" (not shown here) conditions.

\subsection{Triangulation Technique Measurements}

Figure 8 shows ablation depths in enamel under "dry" conditions for the QSP mode as a function of consecutively delivered laser pulses with pulse energy of $300 \mathrm{~mJ}$, as measured with the

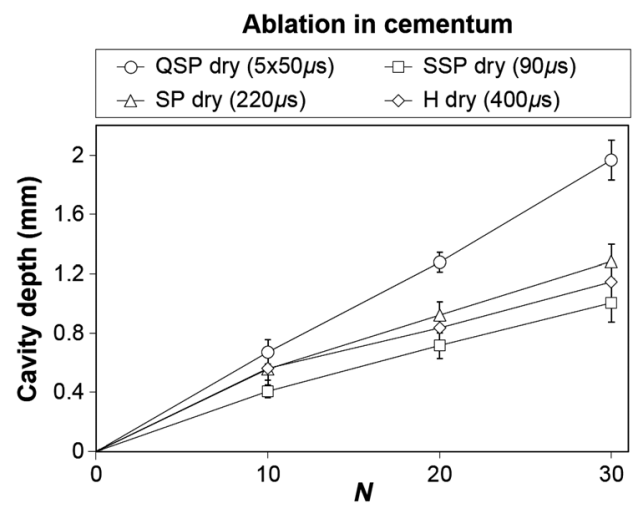

Fig. 7 Measured ablation depths in cementum normalized to the same laser fluence of $F=0.35 \mathrm{~J} / \mathrm{mm}^{2}$, under "dry" conditions for different erbium laser modes. Ablation depths were measured using a focusing optical microscope. 


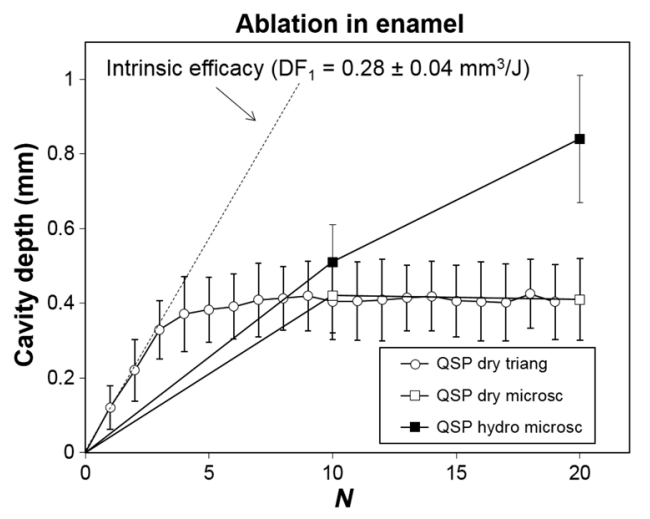

Fig. 8 Er:YAG (QSP) laser ablation depths in enamel for $E_{\text {out }}=$ $300 \mathrm{~mJ}$ under "dry" conditions as measured using a triangulation (circles) and a focusing optical microscope (open squares) technique, and under "hydration" conditions as measured with a focusing optical microscope (full squares) technique. Dashed line represents the intrinsic drilling efficacy in enamel in the absence of desiccation.

triangulation technique. For comparison, the measured ablation depths under "dry" and "hydration" conditions, as obtained using the microscope technique for $N=10$ and 20 pulses, are also shown. As can be seen from Fig. 8, the ablation process starts with a high, "intrinsic" drilling efficacy, represented by the initial slope of the $L$ versus $N$ dependence and then saturates as a result of desiccation.

The intrinsic drilling efficacy $\left(\mathrm{DF}_{1}\right)$ of the QSP mode in enamel under "dry" conditions, as obtained with the triangulation method of $\mathrm{DF}_{1}=0.28 \pm 0.04 \mathrm{~mm}^{3} / \mathrm{J}$, is by a factor of $\gamma=2.2$ larger than the cumulative drilling efficacy $\mathrm{DF}_{10}=$ $0.13 \pm 0.03 \mathrm{~mm}^{3} / \mathrm{J}$, as calculated from the measured depth in enamel under "hydration" conditions following $N=10$ pulses using the microscope technique. Approximately the same factor of $\gamma=2$ was also observed for the SSP mode.

Figure 9 shows ablation depths in cementum under "dry" conditions for the QSP mode as a function of consecutively delivered laser pulses with pulse energy of $200 \mathrm{~mJ}$, as measured with the triangulation technique. For comparison, the measured ablation depths under "dry" and "hydration" conditions, as obtained using the microscope technique for $N=10$ and

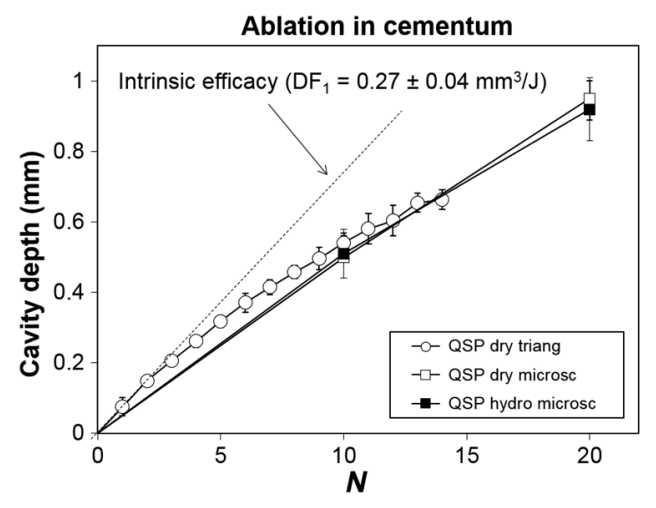

Fig. 9 Er:YAG (QSP) laser ablation depths in cementum for $E_{\text {out }}=$ $200 \mathrm{~mJ}$ under "dry" conditions as measured using a triangulation (circles) and a focusing optical microscope (open squares) technique, and under "hydration" conditions as measured with a focusing optical microscope (full squares) technique. Dashed line represents the intrinsic drilling efficacy in cementum in the absence of desiccation.

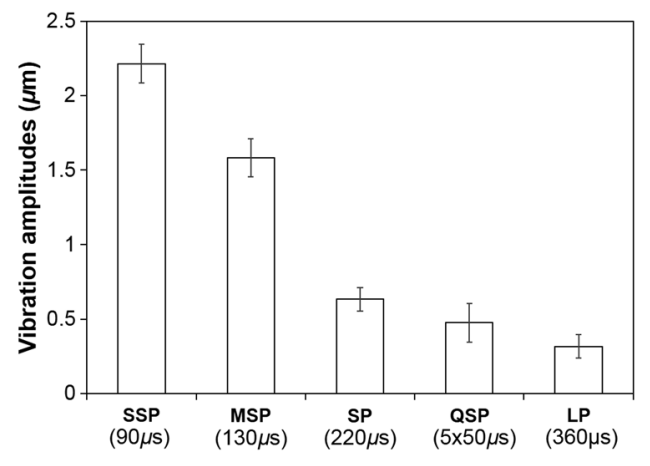

Fig. 10 Measured vibration amplitudes during Er:YAG laser ablation for $E_{\text {out }}=300 \mathrm{~mJ}$ under "hydration" conditions for different pulse modes. The amplitudes were obtained by counting the number of interference fringes before the first change in the direction of movement.

$N=20$ pulses, are also shown. As can be seen from Fig. 9, the ablation process starts with a high, "intrinsic" drilling efficacy, represented by the initial slope of the $h$ versus $N$ dependence, and then saturates as a result of desiccation.

The intrinsic drilling efficacy $\left(\mathrm{DF}_{1}\right)$ of the QSP mode in cementum under "dry" conditions, as obtained with the triangulation method of $\mathrm{DF}_{1}=0.27 \pm 0.04 \mathrm{~mm}^{3} / \mathrm{J}$, is by a factor of $\beta=1.4$ larger than the cumulative drilling efficacy $\mathrm{DF}_{10}=$ $0.20 \pm 0.03 \mathrm{~mm}^{3} / \mathrm{J}$, as calculated from the measured depth in enamel following $N=10$ pulses under "dry" conditions using the microscope technique. Similar comparison for the SSP mode yields approximately the same factor of $\beta=1.4$.

\subsection{Interferometric Vibration Measurements}

Figure 10 shows the measured vibration amplitudes during Er:YAG laser ablation for $E_{\text {out }}=300 \mathrm{~mJ}$ under "hydration" conditions for different pulse modes. The amplitudes were obtained by counting the number of interference fringes before the first change in the direction of movement. Results show that despite having the highest ablation efficacy in enamel (see Fig. 5), the QSP mode results in a significant reduction in vibrations (a decrease by more than a factor of 4 compared with SSP).

\section{Discussion}

Our measurements have demonstrated that by reducing the effects of debris screening using the special "chopped pulse" QSP Er:YAG modality, it is possible to significantly increase the drilling efficacy on hard dental tissues (see Figs. 5, 7, and 10), while at the same time reducing tissue desiccation and vibrations. The observed improvement in drilling efficacy on enamel and cementum, which is in agreement with other studies on enamel, ${ }^{25,44,45}$ is attributed to the reduced absorption and scattering of the incoming laser light on ejected debris during laser ablation. Other studies have shown that when using the QSP mode, the reduced scattering and therefore smaller spreading of the beam also results in sharper and well-defined cuts in hard dental tissue. ${ }^{24,25}$

Of all tested erbium modes, the QSP mode exhibited the largest ablation saturation depth in enamel under "dry" conditions (see Fig. 4), indicating the slowest rate of tissue desiccation when compared to other modes. We assume that tissue desiccation is caused by the residual heat that remains in hard dental tissues after erbium laser irradiation and that a larger ablation 
saturation depth indicates lower heat deposition. The residual heating may be caused by heat diffusion from the ablated area deeper into the tissue ${ }^{18,20,46}$ or by extremely hot, reirradiated ejected tissue that falls back to the ablation spot. ${ }^{47}$ The observed reduction in $L_{\text {sat }}$ toward longer standard pulse duration modes (Fig. 3) is in agreement with previous reports that residual heat deposition is increased for longer laser pulses. ${ }^{20,46} \mathrm{We}$ attribute the increased ablation saturation depth of the QSP mode to a significantly reduced amount of scattering and absorption in the debris cloud as QSPs have sufficient debris settling time between micropulses.

Clinical experience shows that with the QSP mode, the procedures are faster and also quieter than with traditional erbium lasers and are more comfortable for patients. ${ }^{48,49}$ This is in agreement with our observation that in spite of significantly higher drilling efficacy, the vibrations are small with the QSP mode (see Fig. 10).

Referring now to the study by Kang et al., ${ }^{27}$ the existence of hydrokinetic "disruptive forces" was inferred from their observation that with spray, the ablation efficacy of Er,Cr:YSGG laser (Biolase Waterlase) in enamel was "up to twice greater" than without spray. Here, it is important to note that in Ref. 27, the ablation efficacy was determined from the measurement of the ablation volume resulting from 10 consecutive laser pulses. However, as also our study has shown, a considerable stalling of the ablation process occurs during the delivery of 10 pulses under "dry" conditions (see Fig. 7). The ablation on enamel starts with a relatively high intrinsic drilling efficacy and as the enamel gets progressively desiccated the ablation stalls at the ablation saturation depth, $L_{\text {sat }}$. In our experiment, the ablation of the Er,Cr:YSGG laser (H mode) was observed to stop after approximately $N=10$ pulses at $L_{\text {sat }}=0.23 \mathrm{~mm}$ (see Fig. 4) unless it was rehydrated in-between pulses with an external water spray (see Fig. 5). For $N=20$ pulses, the cavity depth under "hydrated" conditions was larger by approximately a factor of 2 than under "dry" conditions. As in Ref. 27, the ablation stalling could be prevented also in our experiments by delivering a continuous water spray to the tooth. However, as can be seen from Figs. 11 and 12, the drilling efficacy under "spray" conditions is lower than under "hydration" conditions. This can be well understood considering the

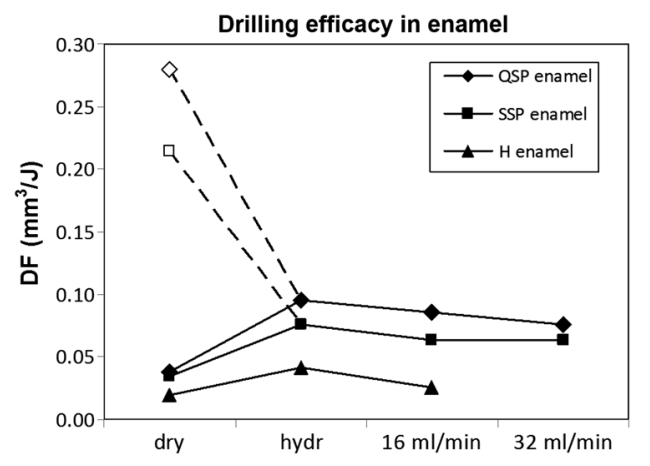

Fig. 11 Ablation drilling efficacies $\left(\mathrm{DF}_{30}\right)$ under "dry," "hydration," "spray $16 \mathrm{ml} / \mathrm{min}$," and "spray $32 \mathrm{ml} / \mathrm{min}$ " conditions for QSP (full diamond), SSP (full square) and $\mathrm{H}$ (full triangle) modalities as obtained from measured depths in enamel using microscope technique following $N=30$ pulses, and intrinsic drilling efficacies $\left(D_{1}\right)$ in enamel under "dry" conditions for QSP (open diamond) and SSP (open square) mode as obtained from initial depth per fluence slopes of the triangulation data.

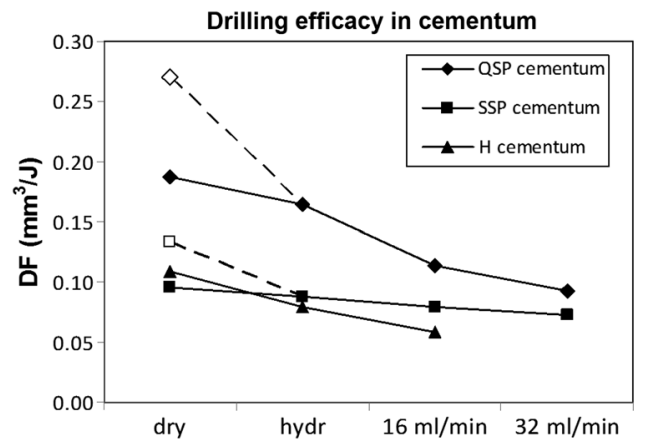

Fig. 12 Ablation drilling efficacies $\left(\mathrm{DF}_{30}\right)$ under "dry," "hydration," "spray $16 \mathrm{ml} / \mathrm{min}$ " and "spray $32 \mathrm{ml} / \mathrm{min}$ " conditions for QSP (full diamond), SSP (full square) and $\mathrm{H}$ (full triangle) modalities as obtained from measured depths in cementum using microscope technique following $N=30$ pulses, and intrinsic drilling efficacies $\left(D_{1}\right)$ in cementum under "dry" conditions for QSP (open diamond) and SSP (open square) modes as obtained from initial depth per fluence slopes of the triangulation data.

absorption coefficient of water for both 2.94 and $2.78 \mu \mathrm{m}$ with absorption lengths of 0.7 and $2 \mu \mathrm{m}$, respectively. Figure 11 shows ablation drilling efficacies $\left(\mathrm{DF}_{30}\right)$ on enamel following $N=30$ pulses under "dry," "hydration," "spray $16 \mathrm{ml} / \mathrm{min}$," and "spray $32 \mathrm{ml} / \mathrm{min}$ " conditions for QSP, SSP, and H modalities, together with the intrinsic drilling efficacies $\left(\mathrm{DF}_{1}\right)$ in enamel under "dry" conditions for QSP and SSP, as obtained from triangulation measurements.

Similarly, Fig. 12 depicts ablation drilling efficacies $\left(\mathrm{DF}_{30}\right)$ on cementum under "dry," "hydration," "spray $16 \mathrm{ml} / \mathrm{min}$," and "spray $32 \mathrm{ml} / \mathrm{min}$ " conditions for QSP, SSP, and H modalities, together with the intrinsic drilling efficacies $\left(\mathrm{DF}_{1}\right)$ in enamel under "dry" conditions for QSP and SSP modes. For enamel and cementum, the intrinsic drilling efficacy is much higher than what is obtained under hydration or water spray conditions. This is in agreement with findings in Ref. 35, where it was concluded that although adding water is required to prevent complete desiccation, any water that is present above the tooth surface in a form of water-spray droplets or on the surface of the tooth in the form of a water layer or pool reduces the ablation efficacy of erbium lasers.

For stationary ablation in one dimension, and negligible scattering and heat diffusion, the crater depth $(L)$ depends on fluence $(F)$ as $L=\left(F-F_{\mathrm{th}}\right) / h_{\mathrm{a}}$, where $h_{\mathrm{a}}$ (in $\left.\mathrm{J} / \mathrm{mm}^{3}\right)$ is the specific heat of ablation. ${ }^{50}$ Therefore, for fluences well above threshold, the specific heat of ablation can be estimated from $h_{\mathrm{a}} \approx F / L=1 / \mathrm{DF}_{1}$. The specific heat of ablation of $h_{\mathrm{a}} \approx$ $4 \mathrm{~J} / \mathrm{mm}^{3}$ for Er:YAG (QSP) ablation of enamel as obtained in this manner from the measured intrinsic drilling efficacy of $\mathrm{DF}_{1}=0.28 \pm 0.4 \mathrm{~mm}^{3} / \mathrm{J}$ compares well with a published estimate of $h_{\mathrm{a}}=5 \pm 1 \mathrm{~J} / \mathrm{mm}^{3}{ }^{18}$ It is interesting to note that for the QSP mode, the intrinsic drilling efficacy $\left(\mathrm{DF}_{1}\right)$ on cementum and enamel is approximately the same. This may not be surprising as the published estimates for specific heat of ablation for enamel (with low water content) and dentin (with higher water content) fall approximately within the same range of values. ${ }^{18,51}$ In addition, the measured drilling efficacy in skin (with very high water content) of $\mathrm{DF}_{1} \approx 0.34 \mathrm{~mm}^{3} / \mathrm{J}^{52}$ gives a similar specific heat of ablation of $h_{\mathrm{a}} \approx 3 \mathrm{~J} / \mathrm{mm}^{3}$.

That is, it is the desiccation, and not the "disruptive forces" of the laser energized water particles, which is responsible for the observed difference in the ablation efficacy under "dry" and 
"hydration" conditions, was further confirmed by our observation that ablation process could be restarted by rehydrating the tooth after being irradiated with 20 pulses under "dry" conditions (see Fig. 3).

We attribute the difference in the effect of desiccation on the ablation efficacy of enamel and cementum to the difference in the water content of both tissues. When untreated, cementum consists of $22 \%_{\text {weight }}$ water, while enamel contains only $3 \%_{\text {weight }}$ water. In order to evaluate the remaining water content in the superficial dental tissue layer following a series of ablative laser pulses, we measured the absorption spectra of the ejected particles from enamel and cementum, which were collected during "dry" ablation on a transparent quartz plate positioned above the ablation surface. The absorption curve of the ejected cementum debris exhibited both the hydroxyapatite (at $2.8 \mu \mathrm{m}$ ) and water (at $2.9 \mu \mathrm{m}$ ) absorption peaks, while the enamel debris exhibited only the hydroxyapatite absorption peak, with no water peak present. The ablation process dries out the enamel, causing the observed saturation of the drilling efficacy in Fig. 3. On the other hand, the superficial cementum layer remains sufficiently hydrated also under consecutive irradiations. ${ }^{31,53,54}$

It is important to note that measured rates of water diffusion in hard tissues are relatively slow. For thin layers of enamel, the effective water diffusion coefficient, $D_{\mathrm{h}}$ has been measured to be $0.510^{-7} \mathrm{~cm}^{2} \mathrm{~s}^{-1} .^{55}$ The diffusion distance $l_{\mathrm{d}}$ to which water diffused into enamel during the hydration time $T=1 \mathrm{~s}$ used in our experiments can be calculated from ${ }^{55}$

$l_{\mathrm{d}}=T D_{\mathrm{h}} \pi^{2} / 4$

The calculated diffusion distance of $l_{\mathrm{d}}=3.5 \mu \mathrm{m}$ is more than 10 times shorter than a typical single-pulse ablation depth under "hydration" conditions. Therefore, the hydration time used not only in this study but also in clinical settings, where higher repetition rates are used, is most probably too short to accomplish a complete rehydration. This was confirmed by two observations in our study. First, when the hydration time was extended from $T=1$ to $10 \mathrm{~s}$, the drilling efficacy for $N=$ 10 SSP pulses increased by $\sim 10 \%$. Even more importantly, as can be seen from Figs. 7 and 10, the measured drilling efficacy on enamel under "hydration" conditions is much smaller than the intrinsic drilling efficacy. This indicates that enamel remained partially desiccated also under "hydration" conditions used in our study.

Finally, it is to be noted that the QSP mode should not be treated as a simple sequence of five individual 50- $\mu$ s-long micropulses. Instead, it is more appropriate to consider the QSP mode sequence as one chopped macropulse. This is because of the optimally chosen temporal spacing of the micropulses, which makes the QSP sequence to behave at least to a certain degree collectively as one macropulse. First, the temporal spacing between the micropulses of $\Delta t_{\mathrm{QSP}} \approx 85 \mu \mathrm{s}$ is significantly shorter than the measured decay time of the dental tissue surface temperature following an ablative Er:YAG laser pulse. Based on the published surface temperature decay curves as measured with a thermal camera, ${ }^{20}$ it can be concluded that during the time $\left(\Delta t_{\mathrm{QSP}}\right)$ in-between micropulses, the surface temperature drops from the tissue ablation temperature remaining at the end of a preceding micropulse by less than 5\%. This means that each subsequent micropulse within the QSP sequence impinges on already preheated superficial tissue layer, making ablation with the QSP micropulse sequence more energy efficient than what would be obtained with five individual micropulses.

Second, the temporal spacing $\Delta t_{\mathrm{OSP}}$ is shorter than the $\mathrm{Er}^{3+}$ ion population inversion time in the YAG crystal of $\sim 300 \mu \mathrm{s}{ }^{56}$ Therefore, a significant share of $\mathrm{Er}^{3+}$ ions remains in their excited laser state following the end of a preceding micropulse and until the start of a subsequent micropulse. Consequently, for each subsequent micropulse, less pumping energy is required to reach lasing threshold, resulting in a significantly higher lasing efficacy of the QSP mode in comparison to the lasing efficiency of five completely independent micropulses. The improved lasing efficiency of every subsequent pulse can be seen also in Fig. 1(c), which shows that the amplitude of micropulses increases from pulse to pulse in a QSP sequence. When a more even distribution of laser intensity during a QSP macropulse is desired, this effect can be compensated by correspondingly increasing the pumping energy for initial micropulse(s).

If we consider, e.g., QSPs being delivered at a repetition rate of $50 \mathrm{~Hz}$, this would result in an emission of 250 micropulses per second. However, as explained above, in terms of the ablation efficacy and lasing efficiency, this would not be equivalent to a laser emitting $50 \mu$ s pulses at a constant repetition rate of $250 \mathrm{~Hz}$.

\section{Conclusions}

In conclusion, our experiments have demonstrated that the interaction of laser light with ejected debris has a considerable influence on ablation characteristics of erbium lasers. By using a special chopped pulse Er:YAG (QSP) mode which was designed to reduce the temporal cross-over of incoming laser radiation with ejected debris, it was possible to significantly increase dental enamel and cementum ablation drilling efficacy and reduce the rate of tissue desiccation. The appropriately timed chopped pulse structure of the QSP mode was shown to result in a high cutting efficacy and low thermal side-effects, a characteristic of short pulses, and in low vibrations and sharp cuts, which is a characteristic of longer pulses.

By measuring ablation depths following each erbium laser pulse, as opposed to measuring cumulative ablation depths following a number of consecutively delivered laser pulses, it was possible to track a progressive reduction in ablation efficacy with the number of delivered laser pulses, which was attributed to a progressive desiccation of dental tissue under erbium laser radiation. It was shown that the initial ("intrinsic") ablation efficacy under "dry" conditions in enamel, and also in cementum, was significantly higher than as would be determined from measured depths following 10 or more laser pulses, regardless of whether the tissue was rehydrated using water spray during laser irradiation experiment. Contrary to a clinical setting where typically the drilling in cementum is observed to be faster than in enamel, the intrinsic drilling efficacy on enamel and on cementum was measured to be approximately the same, in spite of the large difference in water content. It is also concluded that for laser pulse repetition rates of $0.2 \mathrm{~Hz}$ or more, as is typical of clinical settings, adding water to the ablation spot rehydrates the tissue only partially, and especially in enamel, is not sufficient to restore the high initial intrinsic laser drilling efficacy. Additionally, it is concluded, in agreement with previous studies, that there is no evidence for the "hydrokinetic" ablation mechanism, which has been postulated based on the observed difference between the cumulative drilling efficacy of consecutively delivered pulses under "dry" and "spray" conditions. 


\section{Acknowledgments}

Authors wish to thank Fotona (www.fotona.com) and Laser and Health Academy (www.laserandhealthacademy.com) for providing laser systems. The study was funded by the Ministry of Education, Science, Culture, and Sport of Slovenia, and Fotona. Currently, three of the authors (L.N., S.T., and L.M.) are also employees of Fotona.

\section{References}

1. J. Meister et al., "Influence of water content in dental enamel and dentin on ablation with erbium YAG and erbium YSGG lasers," J. Biomed. Opt. 11(3), 034030 (2006).

2. R. Hibst, "Lasers for caries removal and cavity preparation: state of the art and future directions," J. Oral Laser Appl. 2, 203-211 (2002).

3. M. Majaron et al., "Thermo-mechanical laser ablation of soft tissue: modeling the micro-explosions," Appl. Phys. B 69, 71-80 (1999).

4. L. J. Walsh, "The current status of laser applications in dentistry," Austr. Dent. J. 48(3), 146-155 (2003).

5. R. Hibst and U. Keller, "Experimental studies of the application of the Er:YAG laser on dental hard substances: I. Measurement of the ablation rate," Lasers Surg. Med. 9(4), 338-344 (1989).

6. J. T. Walsh, T. J. Flotte, and T. F. Deutsch, "Er:YAG laser ablation of tissues: effect of pulse duration and tissue type on thermal damage," Lasers Surg. Med. 9(4), 314-326 (1989).

7. U. Keller and R. Hibst, "Experimental studies of the application of the Er:YAG laser on dental hard substances: II Light microscopic and SEM investigations," Lasers Surg. Med. 9(4), 345-351 (1989).

8. W. Seka et al., "Laser ablation of hard dental tissues from explosive ablation to plasma mediated ablation," Proc. SPIE 2672, 144-158 (1996).

9. E. L. Tanzi and T. S. Alster, "Single-pass carbon dioxide versus multiplepass Er:YAG laser skin resurfacing: a comparison of postoperative wound healing and side-effect rates," Dermatol. Surg. 29(1), 80-84 (2003).

10. S. Stubinger et al., "Er:YAG laser osteotomy: preliminary clinical and histological results of a new technique for contact-free bone surgery," Eur. Surg. Res. 42, 150-156 (2009).

11. M. Packer, "New phacoemulsification technologies," J. Cataract Refract. Surg. 28, 1054-1060 (2002).

12. M. Gambacciani, "Vaginal erbium laser: the second-generation thermotherapy for the genitourinary syndrome of menopause," Climacteric 18, 1-7 (2015).

13. K. Nemes, M. Lukac, and J. Mozina, "Variable square pulse vs conventional PFN pumping of Er:YAG laser," Opt. Laser Technol. 44, 664-668 (2012).

14. I. Anic et al., "Vibrations produced during erbium: yttrium-aluminumgarnet laser irradiation," Lasers Med. Sci. 24, 697-701 (2009).

15. M. Lukac et al., "Optoacoustic effects during Er:YAG laser ablation in hard dental tissues," Proc. SPIE 2327, 93-100 (1994).

16. M. Lukac et al., "Super short VSP Er:YAG pulses for fast and precise cavity preparation," J. Oral Laser Appl. 4, 171-173 (2004).

17. T. Perhavec and J. Diaci, "Comparison of Er:YAG and Er, Cr:YSGG dental lasers," J. Oral Laser Appl. 8, 87-94 (2008).

18. B. Majaron et al., "Heat diffusion and debris screening in Er:YAG laser ablation of hard biological tissues," Appl. Phys. B 66, 479-487 (1998).

19. A. Vogel and V. Venugopalan, "Mechanisms of pulsed laser ablation of biological tissues," Chem. Rev. 103(5), 577-644 (2003).

20. T. Perhavec et al., "Heat deposition of erbium lasers in hard dental tissues," J. Oral Laser Appl. 9(4), 205-212 (2009).

21. K Nahen and A. Vogel, "Acoustic spectroscopy of Er:YAG laser ablation of skin:first results," Proc. SPIE 3254, 218-229 (1998).

22. G. G. Zhegova et al., "Perception of pain of Er_YAG dental caries treatment in adolescents-a clinical evaluation," J. IMAB 20(1), 500-503 (2014).

23. K. Nahen and A. Vogel, "Plume dynamics and shielding by the ablation plume during Er:YAG laser ablation," J. Biomed. Opt. 7(2), 165-178 (2002).

24. N. Gutknecht et al., "A novel quantum square pulse (QSP) mode erbium dental laser," J. Laser Health Acad. 2011(1), 15-21 (2011).

25. M. Lukac et al., "Minimally invasive cutting of enamel with QSP mode Er:YAG laser,' J. Laser Dent. 22(1), 28-35 (2014).
26. M. Lukac, N. Malej Primc, and S. Pirnat, "Quantum square pulse Er:YAG lasers for fast and precise hard dental tissue preparation," J. Laser Health Acad. 2012(1), 14-21 (2012).

27. H. W. Kang, I. Rizoiu, and A. J. Welch, "Hard tissue ablation with a spray-assisted mid-IR laser," Phys. Med. Biol. 52, 7243-7259 (2007).

28. L. Grad et al., "Optoacoustic studies of Er:YAG laser ablation in hard dental tissues," Proc. SPIE 2128, 456-465 (1994).

29. M. Lukač et al., "Optoacoustic effects during Er:YAG laser ablation in hard dental tissues," Proc. SPIE 2327, 93-100 (1994).

30. S. R. Visuri, J. T. Walsh, and H. A. Wigdor, "Erbium laser ablation of dental hard tissue: effect of water cooling," Lasers Surg. Med. 18(3), 294-300 (1996).

31. D. Fried et al., "Mechanism of water augmentation during IR laser ablation of dental enamel," Lasers Surg. Med. 31(3), 186-193 (2002).

32. L. R. Eversole, I. Rizoiu, and A. I. Kimmel, "Pulpal response to cavity preparation by an erbium, chromium:YSGG laser powered by a hydrokinetic system," J. Am. Dent. Assoc. 128(8), 1099-1106 (1997).

33. I. Rizoiu and A. I. Kimmel, "Atomized fluid particles for electromagnetic cutting," U.S. patent 5, 741, 247 (1998).

34. X. Zhang et al., "Influence of water layer thickness on hard tissue ablation with pulsed $\mathrm{CO}_{2}$ laser," J. Biomed. Opt. 17(3), 038003 (2012).

35. L. Kuscer and J. Diaci, "Measurements of erbium laser ablation efficacy in hard dental tissues under different water cooling conditions," J. Biomed. Opt. 18(10), 108002 (2013).

36. E. J. Burkes et al., "Wet versus dry enamel ablation by Er:YAG laser," J. Prosthetic Dent. 67, 847-851 (1992).

37. R. J. Freiberg and C. Cozean, "Pulsed erbium laser ablation of hard dental tissue: the effects of atomized water spray vs water surface film," Proc. SPIE 4610, 74-84 (2002).

38. G. B. Altshuler et al., "Physical aspects of cavity formation of Er-laser radiation," Proc. SPIE 2394, 211-222 (1995).

39. M. Lukac et al., "Influence of water absorption shift on ablation speed of Er:YAG and Er, Cr:YSGG dental lasers," J. Laser Health Acad. 2013(1), 17-22 (2013).

40. J. Arends, J. L. Ruben, and D. Inaba, "Major topics in quantitative microradiography of enamel, and dentin: R parameter, mineral distribution visualization, and hyper-remineralization," Adv. Dent. Res. 11(4), 403-414 (1997).

41. M. Lukac and T. Suhovrsnik, "Comparison of methods for measuring ablation efficacy of erbium dental lasers," J. Laser Health Acad. 2015(1), 8-10 (2015).

42. R. G. Dorsch et al., "Laser triangulation: fundamental uncertainty in distance measurement," Appl. Opt. 33, 1306-1314 (1994).

43. D. Malacara, Optical Shop Testing, 3rd ed., Wiley, Hoboken, New Jersey (2007).

44. N. M. Primc and M. Lukac, "Quantum square pulse mode ablation measurements with digitally controlled Er:YAG dental laser handpiece," J. Laser Health Acad. 2013(1), 1-5 (2013).

45. C. Filipic and T. Suhovrsnik, "Comparative measurement of the ablation efficacy of quantum square pulse Er:YAG dental laser," J. Laser Health Acad. 2013(2), 8-12 (2013).

46. D. Fried, J. Ragadio, and A. Champion, "Residual heat deposition in dental enamel during IR laser ablation at 2.79, 2.94, 9.6, and $10.6 \mu \mathrm{m}$," Lasers Surg. Med. 29, 221-229 (2001).

47. J. Neev et al., "Ultrashort pulse lasers for hard tissue ablation," IEEE J. Sel. Top. Quant. Electron. 2, 790-808 (1996).

48. E. Mironov and Z. Mironova, "Quantum square pulse Er:YAG lasers in clinical practice," Int. Mag. Laser Dent. 3, 34-37 (2012).

49. E. Mironov, "Clinical experience with a Quantum Square Pulse (QSP) Er:YAG laser," J. Laser Health Acad. 2012(1), 80-85 (2012).

50. J. A. Izatt et al., "Ablation of calcified biological tissue using pulsed hydrogen fluoride laser radiation," IEEE J. Quantum Electron. 26, 2261-2270 (1990).

51. B. Majaron et al., "Threshold and efficiency analysis in Er:YAG laser ablation of hard dental tissues," Proc. SPIE 2922, 233-242 (1996).

52. K. Nemes et al., "Dependence of skin ablation depths on Er:YAG laser fluence," J. Laser Health Acad. 2014(1), 7-13 (2014).

53. L. C. Courrol et al., "Spectroscopic study of ejected dental tissue after Er:YAG laser ablation,” J. Lumin. 102, 96-100 (2003).

54. L. Bachmann et al., "Changes in chemical composition and collagen structure of dentine tissue after erbium laser irradiation," Spectrochim. Acta A 61(11-12), 2634-2639 (2005). 
55. G. H. Didbin, "The water in human dental enamel and its diffusional exchange measured by clearance of tritiated water from enamel slabs of varying thickness," Caries Res. 27(2), 81-86 (1993).

56. B. Majaron, T. Rupnik, and M. Lukac, "Temperature and gain dynamics in flashlamp-pumped Er:YAG," IEEE J. Quantum Electron. 32(9), 1636-1996 (1996).

Nejc Lukač is a PhD student at the faculty of mechanical engineering, University of Ljubljana. His main topics of research are the use of lasers and laser pulse shaping in medical applications. He is currently an employee of Fotona, d.o.o.

Tomaž Suhovršnik received his MSc degree in physics from the University of Ljubljana. His main topics of research are the use of lasers and dentistry. He is currently an employee of Fotona, d.o.o.
Matjaž Lukač received his MSc and PhD degrees in laser physics. Since 1986, he has been working as a research associate at the Josef Stefan Institute. His main topics of research are the interaction of laser light with biological tissues and physics of laser sources. Currently, he is also a managing director of Fotona, d.o.o.

Matija Jezeršek is an associate professor for laser applications at the faculty of mechanical engineering, University of Ljubljana. $\mathrm{He}$ is working at the chair of optodynamics and laser applications. He received his $\mathrm{PhD}$ in mechanical engineering from the University of Ljubljana in 2004. His major research interests include three-dimensional optical metrology, laser processing, and research of optodynamic phenomena 